\title{
Maternal one carbon metabolism and arsenic methylation in a pregnancy cohort in Mexico
}

\author{
Jessica E. Laine ${ }^{1}$, Vesna llievski ${ }^{2}$, David B. Richardson ${ }^{1}$, Amy H. Herring ${ }^{3,4}$, Miroslav \\ Stýblo $^{5}$, Marisela Rubio-Andrade ${ }^{6}$, Gonzalo Garcia-Vargas ${ }^{6}$, Mary V. Gamble ${ }^{2}$, and Rebecca \\ C. Fry ${ }^{7}$ \\ ${ }^{1}$ Department of Epidemiology, Gillings School of Global Public Health, University of North \\ Carolina, Chapel Hill, North Carolina, USA \\ ${ }^{2}$ Department of Environmental Health Sciences, Mailman School of Public Health, Columbia \\ University, New York, NY, USA \\ ${ }^{3}$ Department of Biostatistics, Gillings School of Global Public Health, University of North Carolina, \\ Chapel Hill, North Carolina, USA \\ ${ }^{4}$ Carolina Population Center, University of North Carolina, Chapel Hill, North Carolina, USA \\ ${ }^{5}$ Department of Nutrition, Gillings School of Global Public Health, University of North Carolina, \\ Chapel Hill, North Carolina, USA \\ ${ }^{6}$ Facultad de Medicina, Universidad Juarez del Estado de Durango, Gómez Palacio, Durango, \\ Mexico \\ ${ }^{7}$ Department of Environmental Sciences and Engineering, Gillings School of Global Public Health, \\ University of North Carolina, Chapel Hill, North Carolina, USA
}

\section{Abstract}

The prenatal period represents a critical window of susceptibility to inorganic arsenic (iAs) exposure from contaminated drinking water. Ingested iAs undergoes hepatic methylation generating mono and di-methyl arsenicals (MMAs and DMAs, respectively), a process that facilitates urinary arsenic (As) elimination. Differences in pregnant women's metabolism of As as indicated by greater proportions of MMAs and smaller proportions of DMAs in urine are a risk factor for adverse birth outcomes. One carbon metabolism (OCM), the nutritionally-regulated pathway essential for supplying methyl groups, plays a role in As metabolism and is understudied during the prenatal period. In this cross-sectional study from the Biomarkers of Exposure to ARsenic (BEAR) pregnancy cohort in Gómez Palacio, Mexico, we assessed the relationships among OCM indicators (e.g. maternal serum B12, folate, and homocysteine (Hcys)), and levels of iAs and its metabolites in maternal urine and in neonatal cord serum. The prevalence of folate sufficiency (folate levels $>9 \mathrm{nmol} / \mathrm{L}$ ) in the cohort was high 99\%, and hyperhomocysteinemia

Jessica E. Laine jeslaine@email.unc.edu.

Compliance with ethical standards

Conflict of interest The authors declare that they have no conflict of interest.

Electronic supplementary material The online version of this article (https://doi.org/10.1038/s41370-018-0041-1) contains supplementary material, which is available to authorized users. 
(Hcys levels $>10.4 \mu \mathrm{mol} / \mathrm{L}$ ) was low $(8 \%)$. However, $74 \%$ of the women displayed a deficiency in B12 (serum levels $<148 \mathrm{pmol} / \mathrm{L}$ ). Association analyses identified that infants born to mothers in the lowest tertile of serum folate had significantly higher mean levels of \%MMA in cord serum relative to folate replete women. In addition, elevated maternal Hcys was associated with total As in maternal urine and cord serum as well as cord serum \%MMAs. The results from this study indicate that maternal OCM status may influence the distribution of As metabolites in cord serum.

\section{Introduction}

Inorganic arsenic (iAs) continues to contaminate the drinking water of millions of individuals world-wide, representing a critical global public health issue [1]. The main form of arsenic (As) present in drinking water is iAs, where it undergoes hepatic methylation after ingested to generate monomethyl (MMAs) and dimethyl arsenicals (DMAs). This process influences the toxicity of iAs in humans, where differences in individual methylation/ metabolism capacity are associated with increases in risks for diseases. Specifically, higher proportions of MMAs in urine have been associated with, urinary bladder cancer, nonmelanoma skin cancers, carotid atherosclerosis, and chromosomal aberrations (reviewed in [2]). The role of metabolism of iAs in disease etiology may be particularly important during the prenatal period, as it is a critical developmental time with an increase in susceptibility to iAs exposure and toxicity. For example, prenatal iAs exposure has been associated with lower birth weight, preterm birth, reduced height and head circumference, increased susceptibility to infection, and later in life cancers [3]. Additionally, inefficient maternal metabolism of iAs during pregnancy has been associated with decreases in infant birthweight and other birth outcomes $[4,5]$.

There are many factors that may influence methylation/metabolism of iAs including, but not limited to, the level of exposure to iAs via drinking water and/or food [6], age [7], gender [8], pregnancy [9], genotype for arsenic (+3 oxidation state) methyltransferase (AS3MT) [6, $10]$, and nutritional status of an individual $[11,12]$. S-adenosylmethionine (SAM) serves as a methyl donor for the methylation of As, where the synthesis of SAM is influenced by micronutrients involved in one carbon metabolism (OCM), such as folate, cobalamin (vitamin B12), vitamin B6, betaine and choline [13] (Fig. 1). Various biomarkers are used to represent OCM, and potential indicators include, but are not limited to folate, B12, and Hcys in human biological samples [13]. When OCM is compromised due to nutritional deficiencies, Hcys levels become elevated; however, this can be reversed through nutritional supplementation [14].

There is support for the supplementation of OCM nutrients in iAs exposed populations to increase methylation of iAs as indicated by increased \%DMA or higher DMAs/MMAs ratios in urine. For example, the ability of folic acid supplementation to increase iAs methylation in adults exposed to iAs via contaminated drinking water is supported by a recent randomized control trial [12]. Still, the role of OCM nutrients as a modifying factor influencing iAs metabolism during pregnancy is less clear with varied results. In studies carried out in a cross-sectional pregnancy study in Bangladesh, levels of folate and Hcys were associated with methylated iAs metabolites [15]. Similarly, in another cross-sectional 
study in Bangladesh, folate was moderately associated with methylated iAs metabolites [16]. In contrast, in a prospective cohort study in Bangladesh there was no association with any OCM nutrients and methylated iAs metabolites over the course of pregnancy [17]. These differences across studies may be influenced by the levels of iAs, overall nutritional status, or study design. Taken together, there is a need to further characterize the relationship between nutrients involved in OCM and As metabolism during pregnancy.

The primary aim of this cross-sectional study within the Biomarkers of Exposure to ARsenic (BEAR) cohort in Gómez Palacio, Mexico was to examine the levels of maternal serum indicators of OCM (B12, folate, Hcys) in association with biomarkers of iAs exposure and metabolism of iAs during the prenatal period. We hypothesized that OCM indicators of higher B12 and folate, and lower Hcys would be associated with lower \%iAs and \%MMAs, and higher \%DMAs in both maternal and neonatal samples.

\section{Materials And methods}

\section{Cohort selection}

The BEAR cohort was established to investigate the underlying etiologies of health effects associated with prenatal iAs exposure and has been previously described [4]. Briefly, women were recruited prior to the time of delivery from the time frame of August 2011 to March 2012 at the General Hospital of Gómez Palacio. All procedures associated with this study were approved by Institutional Review Boards [4].

A total of 221 women were originally approached for the study, and 93\% $(n=206)$ provided informed consent for participation in the study. Of these, two women were not included based on a confirmation of a twin pregnancy $(n=1 ; 0.5 \%)$, or sample collection failure $(n=$ $1 ; 0.5 \%)$. Additionally, for this cross-sectional analysis there were six women who were not included because there was insufficient serum to measure the OCM indicators. This resulted in a final sample size of 197 women in the present study. For the assessment of neonatal exposure to iAs there were $188 \mathrm{mom} /$ baby pairs, as nine neonates did not have sufficient cord serum samples for analysis.

\section{Determination of exposure to iAs}

Maternal spot urine samples were collected at the hospital between the time of admission and delivery. These were immediately transferred to cryovials and placed in liquid nitrogen. Aliquots of urine samples were shipped on dry ice to UNC-Chapel Hill and immediately stored at $-80^{\circ} \mathrm{C}$. Cord blood was collected after newborn delivery into an anticoagulant-free vacutainer tube. Following clot formation, tubes were centrifuged and serum was collected and stored at $-80{ }^{\circ} \mathrm{C}$ until aliquots were shipped on dry ice to UNC-Chapel Hill, NC, and stored at $-80^{\circ} \mathrm{C}$.

Arsenical levels in maternal urine are used as indicators of maternal and neonatali As exposure representing the prenatal period. Here, concentrations of urinary iAs (U-iAs), urinary MMA (U-MMAs) and DMA (U-DMAs) were determined by HG-AAS with cryotrapping (CT) as described previously [18-20]. Further description of this method is presented in Supplementary Methods. Metabolism efficiency for iAs in maternal urine 
(adjusted for specific gravity) was determined by calculating the percentage of the individual metabolites (iAs, MMAs, DMAs) relative to the total As determined as the sum of U-iAs, UMMAs, and U-DMAs.

Concentrations of neonatal cord serum arsenic included the measurement of cord serumanalyzed iAs (C-iAs), MMAs (C-MMAs), and DMAs (C-DMAs) and were determined using HG-CT-ICP-MS as described previously [19, 21]. Further description of this method is presenting in Supplementary Methods. In the present study, cord serum was used to measure potential neonatal exposure to circulating iAs and As metabolites. To our knowledge cord serum has not been used as a biomarker of As exposure; however, levels of As in serum have been used as a biomarker of As exposure in children and adults [22, 23]. Neonatal cord proportions of As were also calculated based on the percentage of the individual metabolites in neonatal cord serum out of total As (C-tAs) defined as the sum of C-iAs, C-MMAs and CDMAs. The limit of detection levels, and the number of samples below the LOD for all arsenicals are presented in the Supplementary Methods.

\section{Determination of one-carbon metabolism indicators}

Measurements of the indicators for OCM were carried out on banked maternal serum collected between the time of admission and before delivery, that had been stored at $-80{ }^{\circ} \mathrm{C}$, and shipped on dry ice to Columbia University, New York, NY. Maternal serum folate and B12 were analyzed by radio protein-binding assay (SimulTRAC-S; MP Biomedicals, Orangeburg, NY, USA). Hcys levels were analyzed via a HPLC as previously described [24]. OCM indicators for each woman were categorized based on deficiencies, sufficiencies and/or adequate status as has previously been described [25], and as in other iAs-exposed populations [26]. B12 deficiency was set at $<150 \mathrm{pmol}$ and $\geq 150 \mathrm{pmol}$ for B12 sufficiency. Folate deficiency was set at $<9 \mathrm{nmol}$ and $\geq 9 \mathrm{nmol}$ for folate sufficiency. Hyperhomocysteinemia (high Hcys for non-pregnant populations) was defined by Hcys $\geq 10.4 \mathrm{nmol}$ and normal Hcys was set at $<10.4 \mathrm{nmol}$.

\section{Statistical analyses}

Spearman rank correlations were calculated between the levels of arsenicals in both maternal urine and in neonatal cord serum. Spearman rank correlations were also calculated between the levels of arsenicals in both maternal urine and in neonatal cord serum and the OCM indicators in maternal serum, as well as for the individual components of the OCM indicators to one other.

Differences in the levels of the arsenicals based on nutritional categories were calculated to compare those that were B12 sufficient or deficient, those who had high Hcys or normal levels of Hcys using Wilcoxon Rank Sum tests, and between tertiles of folate for those in the lowest tertile ( $<29.0 \mathrm{nmol}$ ), middle tertile ( $\geq 29.0 \mathrm{nmol}$ and $<47.0 \mathrm{nmol}$ ) or the upper tertile ( $\geq 47.0 \mathrm{nmol}$ ) using an ANOVA. The same comparisons for individual OCM indicators by nutritional categories were calculated. However, comparisons were not made for those who were deficient/sufficient in folate as there were not enough women whose serum levels indicated a folate deficiency for categorical comparisons. Significance was assessed at $p<0.05$. 
The relationship between maternal biomarkers of B12, folate, and Hcys and total arsenic in maternal urine (U-tAs) and/or neonatal serum (C-tAs) was investigated using adjusted linear regression models. To improve model fit, the independent variables (OCM indicators) and dependent variables (U-tAs, C-tAs) were log transformed. Model fit was assessed by inspecting the normality of residuals. Regression assumptions of linearity and the homogeneity of residuals were evaluated by examination of appropriate residual plots. Beta regression models were used to examine the relationships among the percentages of iAs, MMAs, and DMAs and OCM indicators using a previously established SAS macro [27]. Beta regression modeling has been previously used for percentage-scaled dependent variables as it captures various skewed unimodal and bimodal distributions [27]. Because both metabolism of iAs and OCM may be influenced by the exposure levels of iAs [16], beta regression models were also conducted in a stratified manner using median levels of U-tAs (lower U-tAs < median of $23.4 \mu \mathrm{g} / \mathrm{L}$; higher U-tAs $\geq$ median of $23.4 \mu \mathrm{g} / \mathrm{L}$ ) as part of a sensitivity analysis. Regression coefficients were converted to a percentage and results are presented as the estimated percent difference in the log odds of \%iAs, \%MMA, and/or $\%$ DMAs with a one unit difference in B12, folate, and/or Hcys. A set of potential confounders for all models was identified a priori based on their known or potential association with both the exposure (the individual OCM indicators) and the outcome (iAs or its methylated metabolites), using a directed acyclic graph. To determine if maternal OCM indicators predicted maternal U-tAs and/or metabolism and neonatal levels of As, all models were adjusted for maternal age (linear continuous), maternal education (categorical), maternal parity (categorical) and gestational age (linear continuous). Significance was assessed using 95\% confidence intervals (CI) that did not include the null value.

\section{Results}

\section{Demographic characteristics}

Selected characteristics of participants in the BEAR cohort are presented in Table 1. Full details of the study and the cohort participants have been previously described [4]. Briefly, all women were Hispanic and were an average age of 24 years at the time of delivery. The majority of women had an educational level of high school or greater (74\%). Almost all of the women reported taking prenatal vitamins during pregnancy, a factor that may influence the levels of OCM (96\%), few reported eating seafood, which is informative for other potential sources of arsenic (22\%) and for the majority of the women this was not their first pregnancy $(66 \%)$.

\section{Exposure to iAs}

The levels of the arsenicals measured in drinking water, maternal urine, and neonatal cord serum are presented in Table 2. The mean level of iAs measured in drinking water (DW-iAs) was $24.7 \mu \mathrm{g} / \mathrm{L}$ (median: 14.0; range: $0.33-235.6$ ). The mean level of specific gravity adjusted maternal total As in urine (U-tAs) was $37.5 \mu \mathrm{g} / \mathrm{L}$ (median: 23.4; range: 4.3-319.7). DW-iAs was significantly positively correlated with U-tAs $\left(r_{\mathrm{S}}=0.60 ; p<0.001\right)$ (see Supplementary Table 1). The average proportions of maternal urinary arsenic metabolites were $6.1 \%$ (range: $0.77-45.1 \%$ ) for \% U-iAs, $6.4 \%$ (range: $1.3-24.9 \%$ ) for \%U-MMAs, 
87.6\% (range: $32.7-96.7 \%$ ) for \%U-DMAs. To facilitate comparisons to other studies, the ratios of U-MMAs/iAs and U-DMAs/MMAs are presented in Table 2.

The average level of neonatal total cord serum As (C-tAs) was $0.36 \mu \mathrm{g} / \mathrm{L}$ (range: 0.0042 3.0). The average proportions were $10.9 \%$ (range: $0.088-69.5$ ) for $\% \mathrm{C}-\mathrm{iAs}, 16.7 \%$ (range: 0.032-51.8) for \%C-MMAs and 72.5\% (range: 21.6-97.8) for \%C-DMAs. To enable comparisons to other studies, the ratios of C-MMAs/iAs and C-DMAs/MMAs are presented in Table 2.

Maternal U-tAs and the proportions of the maternal urinary arsenicals were compared to levels and proportions of the neonatal serum arsenicals using spearman rank correlation calculations $\left(\mathrm{r}_{\mathrm{s}}\right.$ ) (Supplementary Table 1 ). Maternal U-tAs was significantly positively correlated with C-tAs $\left(r_{\mathrm{S}}=0.60 ; p<0.001\right)$. The percentages and ratios of metabolites $\% \mathrm{U}$ MMAs and \%C-MMAs were significantly positiviely correlated $\left(r_{\mathrm{S}}=0.34 ; p<0.001\right)$ as were $\% \mathrm{U}$-DMAs and $\% \mathrm{C}$-DMAs $\left(\mathrm{r}_{\mathrm{S}}=0.17 ; p=0.02\right)$. However, $\% \mathrm{U}$-iAs was not significantly correlated with $\% \mathrm{C}$-iAs, $\left(r_{\mathrm{S}}=0.075 ; p=0.3\right)$. The average proportions of iAs and MMAs were higher in neonatal cord serum $(10.9 \%$ and $16.7 \%$, respectively) compared to maternal urinary samples of iAs and MMAs (6.1\% and 6.4\%, respectively), whereas the percentages of DMAs were lower in neonatal cord serum (72.5\%) in comparison to maternal urine $(87.6 \%)$ (Table 2).

\section{One carbon metabolism indicators}

The levels of maternal serum nutritional OCM indicators are presented in Table 2. The average maternal serum folate level was $40.6 \mathrm{nmol} / \mathrm{L}$ (range: $7.1-171.5$ ). The average maternal serum B12 level was $127.4 \mathrm{pmol} / \mathrm{L}$ (range: 48.0-284.1). The average maternal serum Hcys level was $6.9 \mu \mathrm{mol} / \mathrm{L}$ (range: $4.1-19.4$ ). B12 was not significantly correlated with folate $\left(r_{\mathrm{S}}=-0.021 ; p=0.7\right)$, nor with Hcys $\left(r_{\mathrm{S}}=-0.037 ; p=0.6\right)$. Folate was significantly negatively correlated with Hcys $\left(r_{\mathrm{S}}=-0.25 ; p=0.0005\right)$ (Supplementary Table $3)$.

The levels of individual OCM indicators for each nutritional category are presented in Supplementary Table 3. A large proportion $(n=145,74 \%)$ of the pregnant women were deficient in B12 (Supplementary Table 3). The majority of women $(n=195,99 \%)$ were sufficient in folate, and only two women (1\%) were deficient in folate (data not shown). Only 15 women (8\%) had hyperhomocysteinemia (Supplementary Table 3). As higher levels of Hcys are associated with folate/B12 deficiencies, differences of Hcys by B12 deficiencies and/or tertiles of folate were explored. There was no significant difference in the levels of Hcys by B12 deficiency status, however there was a significant difference in Hcys levels ( $p$ $<0.05$ ) by tertiles of folate. Specifically, individuals in the lowest folate tertile had the highest mean level of Hcys (Supplementary Table 3).

\section{Relationship among OCM indicators and the methylated iAs arsenicals}

Maternal urinary total As (U-tAs) was significantly negatively correlated with B12 $\left(r_{\mathrm{S}}=\right.$ $-0.22 ; p=0.002)$, and positively correlated with Hcys $\left(r_{\mathrm{s}}=0.19 ; p=0.19\right)$ (see

Supplementary Table 2). There were no significant correlations between urinary markers of maternal metabolism (\% U-iAs, \%U-MMAs, and \%U-DMAs) and OCM indicators. 
However, the percentages of cord serum iAs were significantly positively correlated with maternal levels of $\mathrm{B} 12\left(\mathrm{r}_{\mathrm{s}}=0.16 ; p=0.02\right)$. The percentages of cord serum MMAs were significantly negatively associated with maternal folate levels $\left(r_{\mathrm{S}}=-0.15 ; p=0.04\right)$ and positively correlated with maternal Hcys levels $\left(r_{\mathrm{S}}=0.17 ; p=0.01\right)$ (see Supplementary Table 2).

Differences in the levels of maternal urinary or neonatal cord serum levels of arsenicals based on categories of maternal serum OCM indicators are presented in Table 3. Levels of U-tAs were significantly $(p<0.05)$ higher in those who were deficient in B12. There were no significant $(p>0.05)$ differences in any of the other arsenicals and categories of B12. $\%$ U-iAs and \%U-MMAs were higher in those that had a high level of Hcys. \%C-MMAs were significantly lower $(p=0.04)$ in the middle and highest tertile compared to the lowest tertile of folate (Table 2). However, there were no other statistically significant differences ( $p$ $<0.05)$ in any of the other arsenicals by tertiles of folate.

Results from multivariable linear regressions models to assess the relationships among the levels of maternal serum one carbon metabolism indicators and maternal urinary and/or neonatal cord serum levels of total iAs (tAs) are presented in Table 4. Because both the independent variables (OCM indicators) and dependent variables (U-tAs, C-tAs) were log transformed, the beta estimates are interpreted as a difference in the percent of U-tAs or CtAs based on a one percent difference of the OCM indicators. Maternal serum B12 was significantly negatively associated with U-tAs, where a one-unit difference in the percentage of B12 was associated with a $-0.56 \%(95 \%$ CI: $-0.86,-0.26)$ decrease in U-tAs. Maternal serum Hcys was also significantly associated with U-tAs, where a one-unit difference in the percentage of Hcys was associated with a $0.44 \%$ (95\% CI: $0.043,0.83)$ increase in U-tAs. Furthermore, maternal serum Hcys levels were significantly associated with C-tAs, where a one-unit difference in the percentage of Hcys was associated with a $0.48 \%$ (95\% CI: 0.054 , 0.91) increase in C-tAs. There were no significant relationships in U-tAs or C-tAs and maternal serum folate. Beta regression models were used to estimate the log difference in arsenicals based on OCM indicators. There were no significant relationships identified between maternal serum B12 and/or folate levels and maternal urinary or neonatal cord serum iAs metabolites. Interestingly, maternal serum Hcys levels were significantly positively associated with neonatal serum \%C-MMAs, where a 4.4\% (95\% CI: $0.89,7.9)$ increase in MMAs with for a one-unit difference in log Hcys.

To determine if exposure levels to iAs influenced the associations among maternal OCM indicators and the proportions of arsenicals in maternal urinary and neonatal cord samples, a sensitivity analysis was carried out (Supplementary Table 4, Supplementary Figure 1). Stratifying by levels of U-tAs, there were no significant associations between any of the OCM indicators and maternal metabolism indicators with either lower or higher U-tAs exposure. However, for those in the stratum of higher U-tAs, there was a positive association with higher $\mathrm{B} 12$ and \%C-MMAs, where a one-unit difference in the percentage of B12 was associated with a $0.26 \%$ in \%C-MMAs (95\% CI: $0.049,0.48)$. In contrast, for those in the lower stratum of U-tAs there was a negative association between B12 and \%CMMAs ( $\beta$ : -0.012 , 95\% CI: $-0.27,0.24)$. Furthermore, the positive association of Hcys to \%C-MMAs was retained, but only in the higher U-tAs exposure group ( $\beta: 6.3,95 \% \mathrm{CI}: 2.5,10.1)$. In the 
lower stratum of U-tAs there was a negative, but not significant, relationship between Hcys and \%C-MMAs ( $\beta:-0.26,95 \%$ CI: $-7.1,6.6)$ (Supplementary Table 4).

\section{Discussion}

Exposure to inorganic arsenic (iAs) during pregnancy is associated with adverse birth outcomes, and has been previously linked to maternal arsenic (As) metabolism/methylation capacity $[4,5]$. An individual's As methylation capacity is influenced by nutrients involved in OCM [12]. Given that there are relatively few studies that have investigated the relationship between multiple indicators of OCM and As metabolism in pregnant women, we examined these relationships in the biomarkers of exposure to arsenic (BEAR) pregnancy cohort. Specifically, we determined the association between levels of OCM indicators (B12, folate, Hcys) in maternal serum with levels and proportions of iAs metabolites in maternal urine and cord serum. In this region, iAs contamination via drinking water is concerning, as levels are of iAs are range up to 236 parts per billion [4]. Two major findings from the study were that infants born to mothers in the lowest tertile of serum folate had significantly higher mean levels of \%MMA in cord serum and elevated maternal Hcys was associated with higher cord serum \%MMAs. Collectively, the results from this study indicate that maternal OCM status may influence the distribution of As metabolites in cord serum, which may be indicative of iAs toxicity.

Intriguing results that were unexpected in the present study were that majority of the pregnant women were folate sufficient, yet B12 deficient. However, in support of these results, high rates of vitamin B12 insufficiency during pregnancy corresponding with a low prevalence of folate deficiency have been identified in other populations [28]. Additionally, a high prevalence of B12 deficiency for women of reproductive age has been observed recently in the study area from a country-wide survey across Mexico [29]. Of concern, impaired vitamin B12 status during pregnancy has been associated with numerous adverse pregnancy outcomes, including intrauterine growth restriction, preterm delivery, and neural tube defects [30, 31]. B12 deficiency has also been linked to later in life health outcomes, including immune function impairment and neurological and cognitive functions in children [32]. Given that B12 is known to influence Hcys and methionine levels [33], we hypothesized that the observed B12 deficiencies would be associated with the levels of the iAs methylated metabolites in the pregnant women. Indeed, elevated levels of Hcys were positively associated with neonatal \%C-MMAs. Additionally, despite few of the women having folate deficiency, infants born to mothers in the lowest tertile of serum folate had significantly higher mean levels of \%C-MMAs as compared to those born to folate replete women in unadjusted comparisons. These data support that maternal nutritional biomarkers involved in OCM are associated with biomarkers of total As for both maternal and neonatal samples (urine and cord serum, respectively) and \%MMAs in neonatal cord serum.

Maternal urinary levels and neonatal cord serum levels of total As and iAs metabolites were found to be significantly correlated, a finding that has not been previously described. Specifically, maternal total urinary arsenic was significantly positively correlated with neonatal total serum arsenic. Additionally, \%MMAs and \%DMAs were significantly positively correlated between maternal urine and neonatal cord serum. Likely due to a 
number of samples below the LOD, this relationship was not observed for \%iAs. The finding that maternal urinary metabolites and neonatal cord serum metabolites are significantly correlated is an indication that maternal-fetal transport of iAs and its metabolites across the placenta does indeed occur [34]. Furthermore, maternal-fetal correlations between iAs in maternal whole blood and neonatal cord blood is supported by others $[15,34,35]$. Together, these findings support that the developing fetus is exposed to arsenic metabolites, which may have consequences for birth and later in life health effects.

The results from the study highlight that $8 \%$ of the women had elevated levels of Hcys. This is concerning, as elevated Hcys is a well-established risk factor several adverse outcomes including, placental abruption, preeclampsia, and adverse pregnancy outcomes [36]. The overall mean observed level of serum Hcys was $6.9 \mu \mathrm{mol} / \mathrm{L}$, a value that is in line with other populations with high rates of vitamin B12 insufficiency [37, 38]. These higher maternal serum levels of Hcys were associated with higher neonatal serum levels of \%MMAs. While similar results have not been previously identified for \%MMAs in neonatal cord serum, in support of our results, plasma Hcys levels have been positively associated with urinary $\%$ MMAs in an adult population in Bangladesh [39]. This relationship is intuitive, as elevated levels of Hcys are associated with a reduction in SAM levels, the important methyl donor for iAs methylation [9]. Levels of maternal folate and Hcys were correlated as expected, however levels of B12 and hcys were not. This is potentially due to pregnancy-induced physiologic changes, such as hemodilution, increased remethylation of Hcys [40], and increased renal excretion [41]. Taken together, our results highlight that maternal Hcys levels may be an important factor that influences the levels and proportions of iAs metabolites in neonates.

The generalizability of the findings from the present study to other studies and populations that have investigated the relationship of OCM to iAs metabolism is complicated by several factors, namely the differences in both dietary patterns and measurements of OCM indicators and differing levels of exposure to As across populations. Specifically, there are many nutrients that may influence OCM and/or the levels of iAs and metabolism that can contribute to the availability of methyl groups ultimately used in SAM biosynthesis including, betaine, choline, riboflavin, and serine, and other OCM indicators such as B6 and B2 [42]. A comprehensive assessment of all OCM indicators has not yet been fully investigated, particularly during the prenatal period. However, several studies have characterized many important relationships between OCM and levels and metabolism of iAs, including the effect of folic acid supplementation on increasing iAs methylation [12], and recently the effects of multiple B vitamins and their interaction with one another on iAs metabolism [42]. Importantly, when comparing the present study to others, is that many of the studies that examined the effects of folic acid supplementation and other OCM indicators on iAs metabolism were conducted in Bangladesh where the levels of iAs exposure are much higher than in the present study. In contrast, in a recent study with low-moderate levels of exposure to As in US American Indian adults, an association between dietary vitamins B2 and B6 and iAs metabolism was observed but this was not the case for B12 or folate [42]. To better address the relationship between OCM and iAs metabolism future studies will need to consider a broader spectrum of multiple nutrients with measurements of both dietary intake and biomarkers of OCM at varying exposure levels of iAs in order to potentially extrapolate 
a dose-response relationship between OCM and iAs metabolism. Additionally, future studies will need to consider interactions between the OCM indicators with one another [42]. Furthermore, the unexpected finding that higher maternal serum levels of B12 are associated with higher \%MMAs in cord serum are likely influenced by the high prevalence of B12 deficiency in the BEAR cohort, and therefore replication is warranted in B12 sufficient populations. Lastly, studies during pregnancy will require special attention in study design for the assessments of nutrients and As, as there are many factors that influence both OCM and iAs metabolism over the course of pregnancy.

This study is not without limitations that may influence our findings. This study is crosssectional, therefore it is difficult to establish directionality and causality of the effects of OCM over the course of pregnancy on metabolism of iAs. In terms of detailing the underlying reasons for sufficiency or deficiency in the BEAR cohort, the sufficiency of folate is more than likely due to prenatal supplementation of folic acid [28] and is supported by the large number $(96 \%)$ of women in the BEAR cohort who reported taking prenatal vitamins. The causes for the B12 deficiency in the BEAR cohort is not known. B12 deficiency is often attributed to a low dietary intake of B12, such as adhering to a vegetarian diet [31]. Other factors that can influence lower levels of vitamin B12 during pregnancy could also be due to biological changes related to pregnancy including, hemodilution, active transport to the fetus, and changes in binding proteins [43]. Women in the BEAR cohort reported taking prenatal vitamins; however, complete dietary assessments are not available. Together these limitations influence the ability to extrapolate the influencing factors for B12 deficiency, and/or folate sufficiency in this population. However, if there is any potential misclassification of the exposure it would more than likely be non-differential, and therefore would not influence the interpretation of our findings. Additionally, other dietary characteristics may influence the levels of As including contributions of As from rice and seafood. While the majority of the women did not consume seafood, we cannot fully disentangle contribution to DMAs with these self-reports. Furthermore, we cannot rule out any potential unmeasured confounding between OCM and iAs biomarkers. Specifically, it has been suggested that maternal body mass index, unavailable in the BEAR cohort, is associated with both OCM metabolism and As metabolism [16]. Lastly, whole blood As was not measured in neonatal or maternal samples, limiting the ability for comparisons to other studies. While levels of As in whole cord blood have been used before as a measurement of potential neonatal exposure [44], the use of cord serum As for a biomarker of exposure has not. Furthermore, the comparison between As and its metabolites in serum and whole blood has not been fully elucidated; however, levels of As in serum may be lower than levels of As in whole blood [45]. The results of this study support that maternal B12 and Hcys are positively associated \%MMAs in cord serum, signifying that replication of this work is needed.

In conclusion, this study is among the first studies to assess B12, folate, and Hcys on As metabolism in a folate sufficient pregnant population in Mexico. The data highlight that pregnant women and their infants are exposed to iAs and that may necessitate further monitoring for B12 deficiencies. The findings from this study elucidate the need to investigate these relationships as they relate to disease etiologies associated with prenatal iAs exposure. 
Maternal serum one carbon metabolism indicators $(n=197)$

\section{Supplementary Material}

Refer to Web version on PubMed Central for supplementary material.

\section{Acknowledgements}

This research was supported by grants from the National Institute of Environmental Health Sciences (P42ES005948, R01-ES019315, and T32-ES07018).

\section{References}

1. Huang L, Wu H, van der Kuijp TJ. The health effects of exposure to arsenic-contaminated drinking water: a review by global geographical distribution. Int J Environ Health Res. 2015;25:432-52. [PubMed: 25365079]

2. Tseng $\mathrm{CH}$. Arsenic methylation, urinary arsenic metabolites and human diseases: current perspective. J Environ Sci Health C Environ Carcinog Ecotoxicol Rev. 2007;25:1-22. [PubMed: 17365340]

3. Naujokas MF, Anderson B, Ahsan H, Aposhian HV, Graziano JH, Thompson C, et al. The broad scope of health effects from chronic arsenic exposure: update on a worldwide public health problem. Environ Health Perspect. 2013;121:295-302. [PubMed: 23458756]

4. Laine JE, Bailey KA, Rubio-Andrade M, Olshan AF, Smeester L, Drobná Z, et al. Maternal arsenic exposure, arsenic methylation efficiency, and birth outcomes in the Biomarkers of Exposure to ARsenic (BEAR) pregnancy cohort in Mexico. Environ Health Perspect. 2015;123:186-92. [PubMed: 25325819]

5. Gilbert-Diamond D, Emond JA, Baker ER, Korrick SA, Karagas MR. Relation between in utero arsenic exposure and birth outcomes in a cohort of mothers and their newborns from New Hampshire. Environ Health Perspect. 2016;124:1299-307. [PubMed: 26955061]

6. Jansen RJ, Argos M, Tong L, Li J, Rakibuz-Zaman M, Islam MT, et al. Determinants and consequences of arsenic metabolism efficiency among 4794 individuals: demographics, lifestyle, genetics, and toxicity. Cancer Epidemiol Biomark Prev. 2016;25:381-90.

7. Rodrigues EG, Kile M, Hoffman E, Quamruzzaman Q, Rahman M, Mahiuddin G, et al. GSTO and AS3MT genetic polymorphisms and differences in urinary arsenic concentrations among residents in Bangladesh. Biomarkers. 2012;17:240-7. [PubMed: 22339537]

8. Lindberg AL, Kumar R, Goessler W, Thirumaran R, Gurzau E, Koppova K, et al. Metabolism of low-dose inorganic arsenic in a central European population: influence of sex and genetic polymorphisms. Environ Health Perspect. 2007;115:1081-6. [PubMed: 17637926]

9. Vahter M Mechanisms of arsenic biotransformation. Toxicology.2002;181-2:211-7. [PubMed: 11893417]

10. Karagas MR, Stukel TA, Morris JS, Tosteson TD, Weiss JE, Spencer SK, et al. Skin cancer risk in relation to toenail arsenic concentrations in a US population-based case-control study. Am J Epidemiol. 2001;153:559-65. [PubMed: 11257063]

11. Vahter ME. Interactions between arsenic-induced toxicity and nutrition in early life. J Nutr. 2007;137:2798-804. [PubMed: 18029502]

12. Peters BA, Hall MN, Liu X, Parvez F, Sanchez TR, van Geen A, et al. Folic acid and creatine as therapeutic approaches to lower blood arsenic: a randomized controlled trial. Environ Health Perspect. 2015;123:1294-301. [PubMed: 25978852]

13. Hall MN, Gamble MV. Nutritional manipulation of one-carbon metabolism: effects on arsenic methylation and toxicity. J Toxicol. 2012;2012:595307. [PubMed: 22523489]

14. Swanson DA, Liu ML, Baker PJ, Garrett L, Stitzel M, Wu J, et al. Targeted disruption of the methionine synthase gene in mice. Mol Cell Biol. 2001;21:1058-65. [PubMed: 11158293] 
15. Hall M, Gamble M, Slavkovich V, Liu X, Levy D, Cheng Z, et al. Determinants of arsenic metabolism: blood arsenic metabolites, plasma folate, cobalamin, and homocysteine concentrations in maternal-newborn pairs. Environ Health Perspect. 2007;115: 1503-9. [PubMed: 17938743]

16. Li L, Ekström EC, Goessler W, Lönnerdal B, Nermell B, Yunus M, et al. Nutritional status has marginal influence on the metabolism of inorganic arsenic in pregnant Bangladeshi women. Environ Health Perspect. 2008;116:315-21. [PubMed: 18335097]

17. Gardner RM, Nermell B, Kippler M, Grandér M, Li L, Ekström EC, et al. Arsenic methylation efficiency increases during the first trimester of pregnancy independent of folate status. Reprod Toxicol. 2011;31:210-8. [PubMed: 21078382]

18. Devesa V, Maria Del Razo L, Adair B, Drobna Z, Waters SB, Hughes MF, et al. Comprehensive analysis of arsenic metabolites by $\mathrm{pH}$-specific hydride generation atomic absorption spectrometry. J Anal At Spectrom. 2004;19:1460-7.

19. Hernandez-Zavala A, Matousek T, Drobna Z, Paul DS, Walton F, Adair BM, et al. Speciation analysis of arsenic in biological matrices by automated hydride generation-cryotrapping-atomic absorption spectrometry with multiple microflame quartz tube atomizer (multiatomizer). J Anal Spectrom. 2008;23:342-51.

20. Hernandez-Zavala A, Drobna Z, Styblo M, Thomas DJ. Analysis of arsenical metabolites in biological samples. Curr Protoc Toxicol. 2009;42:4.33. 31-34.33.17.

21. Matoušek T, Currier JM, Trojánková N, Saunders RJ, Ishida MC, González-Horta C, et al. Selective hydride generation-cryotrapping- ICP-MS for arsenic speciation analysis at picogram levels: analysis of river and sea water reference materials and human bladder epithelial cells. $\mathrm{J}$ Anal Spectrom. 2013;28:1456-65.

22. Fukai Y, Hirata M, Ueno M, Ichikawa N, Kobayashi H, Saitoh H, et al. Clinical pharmacokinetic study of arsenic trioxide in an acute promyelocytic leukemia (APL) patient: speciation of arsenic metabolites in serum and urine. Biol Pharm Bull. 2006;29: 1022-7. [PubMed: 16651738]

23. Skalny AV, Simashkova NV, Klyushnik TP, Grabeklis AR, Radysh IV, Skalnaya MG, et al. Assessment of serum trace elements and electrolytes in children with childhood and atypical autism. J Trace Elem Med Biol. 2017;43:9-14. [PubMed: 27707611]

24. Gamble MV, Ahsan H, Liu X, Factor-Litvak P, Ilievski V, Slavkovich V, et al. Folate and cobalamin deficiencies and hyperhomocysteinemia in Bangladesh. Am J Clin Nutr. 2005;81: 1372-7. [PubMed: 15941889]

25. de Benoist B Conclusions of a WHO Technical Consultation on folate and vitamin B12 deficiencies. Food Nutr Bull. 2008;29: S238-44. [PubMed: 18709899]

26. Howe CG, Niedzwiecki MM, Hall MN, Liu X, Ilievski V, Slavkovich V, et al. Folate and cobalamin modify associations between $\mathrm{S}$-adenosylmethionine and methylated arsenic metabolites in arsenic-exposed Bangladeshi adults. J Nutr. 2014;144: 690-7. [PubMed: 24598884]

27. Swearinge CJ, Melguizo Castro MS, Bursac Z. Modeling percentage outcomes: the \%beta_regression macro. In proceedings of the SAS Global Forum, Las Vegas NV, 2011, paper 335:1-12.

28. Sukumar N, Rafnsson SB, Kandala NB, Bhopal R, Yajnik CS, Saravanan P. Prevalence of vitamin B-12 insufficiency during pregnancy and its effect on offspring birth weight: a systematic review and meta-analysis. Am J Clin Nutr. 2016;103:1232-51. [PubMed: 27076577]

29. Shamah-Levy T, Villalpando S, Mejía-Rodríguez F, Cuevas-Nasu L, Gaona-Pineda EB, RangelBaltazar E, et al. Prevalence of iron, folate, and vitamin B12 deficiencies in 20 to 49 years old women: Ensanut 2012. Salud Publica Mex. 2015;57:385-93. [PubMed: 26544999]

30. Ray JG, Goodman J, O’Mahoney PR, Mamdani MM, Jiang D. High rate of maternal vitamin B12 deficiency nearly a decade after Canadian folic acid flour fortification. QJM. 2008;101:475-7. [PubMed: 18337285]

31. Folate Refsum H., vitamin B12 and homocysteine in relation to birth defects and pregnancy outcome. Br J Nutr. 2001;85(Suppl 2):S109-13. [PubMed: 11509098]

32. Molloy AM, Kirke PN, Brody LC, Scott JM, Mills JL. Effects of folate and vitamin B12 deficiencies during pregnancy on fetal, infant, and child development. Food Nutr Bull. 2008;29: S101-111. discussionS112-05 [PubMed: 18709885] 
33. Shane B, Stokstad EL. Vitamin B12-folate interrelationships. Annu Rev Nutr. 1985;5:115-41. [PubMed: 3927946]

34. Concha G, Vogler G, Lezcano D, Nermell B, Vahter M. Exposure to inorganic arsenic metabolites during early human development. Toxicol Sci. 1998;44:185-90. [PubMed: 9742656]

35. Environmental Protection Agency (EPA). Fact sheet: Drinking Water Standard for arsenic. Washington, DC: Office of Water, U. S. Environmental Protection Agency; 2001.

36. Forges T, Monnier-Barbarino P, Alberto JM, Guéant-Rodriguez RM, Daval JL, Guéant JL. Impact of folate and homocysteine metabolism on human reproductive health. Hum Reprod Update. 2007;13:225-38. [PubMed: 17307774]

37. Park H, Kim YJ, Ha EH, Kim KN, Chang N. The risk of folate and vitamin B(12) deficiencies associated with hyperhomocysteinemia among pregnant women. Am J Perinatol. 2004;21: 469-75. [PubMed: 15580543]

38. Yajnik CS, Deshpande SS, Jackson AA, Refsum H, Rao S, Fisher DJ, et al. Vitamin B12 and folate concentrations during pregnancy and insulin resistance in the offspring: the Pune Maternal Nutrition Study. Diabetologia. 2008;51:29-38. [PubMed: 17851649]

39. Gamble MV, Liu X, Ahsan H, Pilsner R, Ilievski V, Slavkovich V, et al. Folate, homocysteine, and arsenic metabolism in arsenicexposed individuals in Bangladesh. Environ Health Perspect. 2005;113:1683-8. [PubMed: 16330347]

40. Steegers-Theunissen RP, Wathen NC, Eskes TK, van Raaij-Selten B, Chard T. Maternal and fetal levels of methionine and homocysteine in early human pregnancy. Br J Obstet Gynaecol. 1997;104:20-24. [PubMed: 8988690]

41. Cikot RJ, Steegers-Theunissen RP, Thomas CM, de Boo TM, Merkus HM, Steegers EA. Longitudinal vitamin and homocysteine levels in normal pregnancy. Br J Nutr. 2001;85:49-58. [PubMed: 11227033]

42. Spratlen MJ, Gamble MV, Grau-Perez M, Kuo CC, Best LG, Yracheta J, et al. Arsenic metabolism and one-carbon metabolism at low-moderate arsenic exposure: Evidence from the Strong Heart Study. Food Chem Toxicol. 2017;105:387-97. [PubMed: 28479390]

43. Koebnick C, Heins UA, Dagnelie PC, Wickramasinghe SN, Ratnayaka ID, Hothorn T, et al. Longitudinal concentrations of vitamin $\mathrm{B}(12)$ and vitamin $\mathrm{B}(12)$-binding proteins during uncomplicated pregnancy. Clin Chem. 2002;48:928-33. [PubMed: 12029010]

44. Hall M, Chen Y, Ahsan H, Slavkovich V, van Geen A, Parvez F, et al. Blood arsenic as a biomarker of arsenic exposure: results from a prospective study. Toxicology. 2006;225:225-33. [PubMed: 16860454]

45. Kingsley G, Schaffert R, Harris WW. Microdetermination of arsenic and its application to biological material. Anal Chem. 1951;23:914-9. 


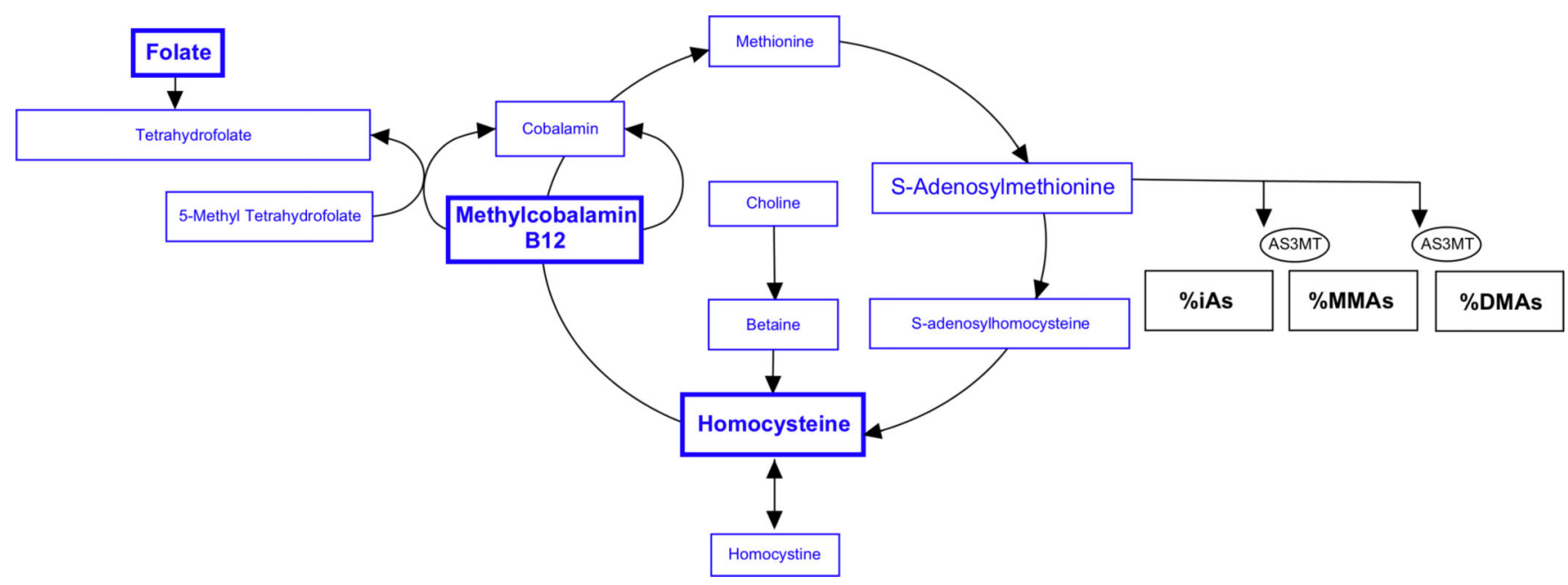

Fig. 1.

Overview of one-carbon metabolism (OCM) (highlighted in blue) and arsenic (As) metabolism (highlighted in black) with emphasis on measurements from the present study within the Biomarkers to ARsenic (BEAR) cohort. OCM indicators, measured in maternal serum in BEAR, are indicated by the bolded blue boxes and include, folate,

methylcobalamin (B12), and Homocysteine (Hcys). Reactions that influence As metabolism are indicated by dietary folates that are reduced to tetrahydrofolate (THF) in a reaction catalyzed by B12, where the methyl group of 5-methyl-THF is transferred to homocysteine, generating methionine and regenerating THF. Methionine adenosyltransferase activates methionine to form $\mathrm{S}$-adenosylmethionine (SAM). SAM serves as a universal methyl donor for numerous reactions, including methylation of As, along with arsenic (+3) methy transferase (AS3MT). Here As is reduced and methylated, resulting in proportions of arsenicals (out of the total As), measured in maternal urine and neonatal cord serum in BEAR, and include \%iAs (unmetabolized iAs) \%MMAs (mono-methyl As), and \%DMAs (dimethyl As). The byproduct of these methylation reactions, S-adenosylhomocysteine $(\mathrm{SAH})$, is hydrolyzed to generate homocysteine. SAH is a potent inhibitor of most SAMdependent methylations. Homocysteine is either used to regenerate methionine or is directed to the transsulfuration pathway and is ultimately catabolized 


\section{Table 1}

Selected demographic characteristics of participants of the biomarkers of exposure to arsenic (BEAR)

\begin{tabular}{|c|c|}
\hline Characteristic & Total $(n=197)$ \\
\hline & $N(N \%)$ \\
\hline Maternal age at delivery, years ${ }^{a}$ & $24(5.5)$ \\
\hline Race/ethnicity (Hispanic) & $197(100)$ \\
\hline \multicolumn{2}{|l|}{ Educational level } \\
\hline$<$ High School & $51(25.9)$ \\
\hline High School & $95(48.2)$ \\
\hline$>$ High School & $51(25.9)$ \\
\hline \multicolumn{2}{|l|}{ Smoking Status } \\
\hline Non-smokers & $184(93.4)$ \\
\hline Current smokers & $13(6.6)$ \\
\hline \multicolumn{2}{|l|}{ Alcohol consumption } \\
\hline None & $156(79.2)$ \\
\hline Some & $41(20.8)$ \\
\hline \multicolumn{2}{|l|}{ Daily prenatal vitamin intake } \\
\hline No & $8(4.1)$ \\
\hline Yes & $189(95.9)$ \\
\hline \multicolumn{2}{|l|}{ Seafood consumption } \\
\hline None & $154(78.2)$ \\
\hline Some & $43(21.8)$ \\
\hline \multicolumn{2}{|l|}{ Parity } \\
\hline 1st pregnancy & $68(34.5)$ \\
\hline More than 1 pregnancy & $129(65.5)$ \\
\hline Gestational age (weeks) $^{a}$ & $39.3(1.2)$ \\
\hline \multicolumn{2}{|l|}{ Newborn sex $(n=188)$} \\
\hline Male & $103(52.3)$ \\
\hline Female & $94(47.7)$ \\
\hline Birth weight $(\mathrm{g})(n=188)$ & $3338.3(481.7)$ \\
\hline
\end{tabular}




\section{Table 2}

Levels of arsenicals and one carbon metabolism indicators from participants ${ }^{a}$ of the biomarkers of exposure to arsenic (BEAR) cohort

\begin{tabular}{|c|c|c|c|}
\hline & Mean & Median & Range \\
\hline DW-iAs $(\mu \mathrm{g}$ As/L) $(n=197)$ & 24.7 & 14.0 & $0.33-235.6$ \\
\hline \multicolumn{4}{|c|}{ Maternal urinary arsenicals ${ }^{b}(n=197)$} \\
\hline U-tAs $(\mu \mathrm{g} / \mathrm{L})$ & 37.5 & 23.4 & $4.3-319.7$ \\
\hline $\mathrm{U}$-iAs $(\mu \mathrm{g} / \mathrm{L})$ & 2.1 & 1.3 & $0.14-23.0$ \\
\hline U-MMAs $(\mu \mathrm{g} / \mathrm{L})$ & 2.3 & 1.3 & $0.082-18.2$ \\
\hline U-DMAs $(\mu \mathrm{g} / \mathrm{L})$ & 33.1 & 20.8 & $1.4-292.5$ \\
\hline U-iAs $(\%)$ & 6.1 & 5.3 & $0.77-45.1$ \\
\hline U-MMAs (\%) & 6.4 & 5.9 & $1.3-24.9$ \\
\hline U-DMAs $(\%)$ & 87.6 & 88.5 & $32.7-96.7$ \\
\hline U-MMAs/iAs & 1.2 & 1.2 & $0.13-5.5$ \\
\hline U-DMAs/MMAs & 17.3 & 14.7 & $1.5-75.2$ \\
\hline \multicolumn{4}{|c|}{ Neonatal cord serum arsenicals $(n=188)$} \\
\hline C-tAs $(\mu \mathrm{g} / \mathrm{L})$ & 0.36 & 0.24 & $0.0042-3.0$ \\
\hline $\mathrm{C}$-iAs $(\mu \mathrm{g} / \mathrm{L})$ & 0.038 & 0.011 & $0.00085-0.50$ \\
\hline C -MMAs $(\mu \mathrm{g} / \mathrm{L})$ & 0.062 & 0.044 & $0.000042-0.56$ \\
\hline C -DMAs $(\mu \mathrm{g} / \mathrm{L})$ & 0.26 & 0.17 & $0.0031-2.7$ \\
\hline $\mathrm{C}$-iAs (\%) & 10.9 & 4.5 & $0.088-69.5$ \\
\hline C-MMAs (\%) & 16.7 & 16.7 & $0.032-51.8$ \\
\hline C -DMAs (\%) & 72.5 & 74.9 & $21.6-97.8$ \\
\hline C-MMAs/iAs & 21.0 & 3.9 & $0.00075-249.4$ \\
\hline C-DMAs/MMAs & 23.3 & 4.5 & $0.92-1768.1$ \\
\hline \multicolumn{4}{|c|}{ Maternal serum one carbon metabolism indicators ${ }^{b}(n=197)$} \\
\hline Folate $(\mathrm{nmol} / \mathrm{L})$ & 40.6 & 37.9 & $7.1-171.5$ \\
\hline $\mathrm{B} 12(\mathrm{pmol} / \mathrm{L})$ & 127.4 & 116.5 & $48.0-284.1$ \\
\hline Hcys ( $\mu \mathrm{mol} / \mathrm{L})$ & 6.9 & 6.4 & $4.1-19.4$ \\
\hline
\end{tabular}

${ }^{a}$ Reported results are based upon a sample of 197 and 188, for measured values for urinary and neonatal cord serum arsenicals, respectively and 197 samples of maternal OCM indicators.

${ }^{b}$ Maternal urinary arsenicals were adjusted by specific gravity. 


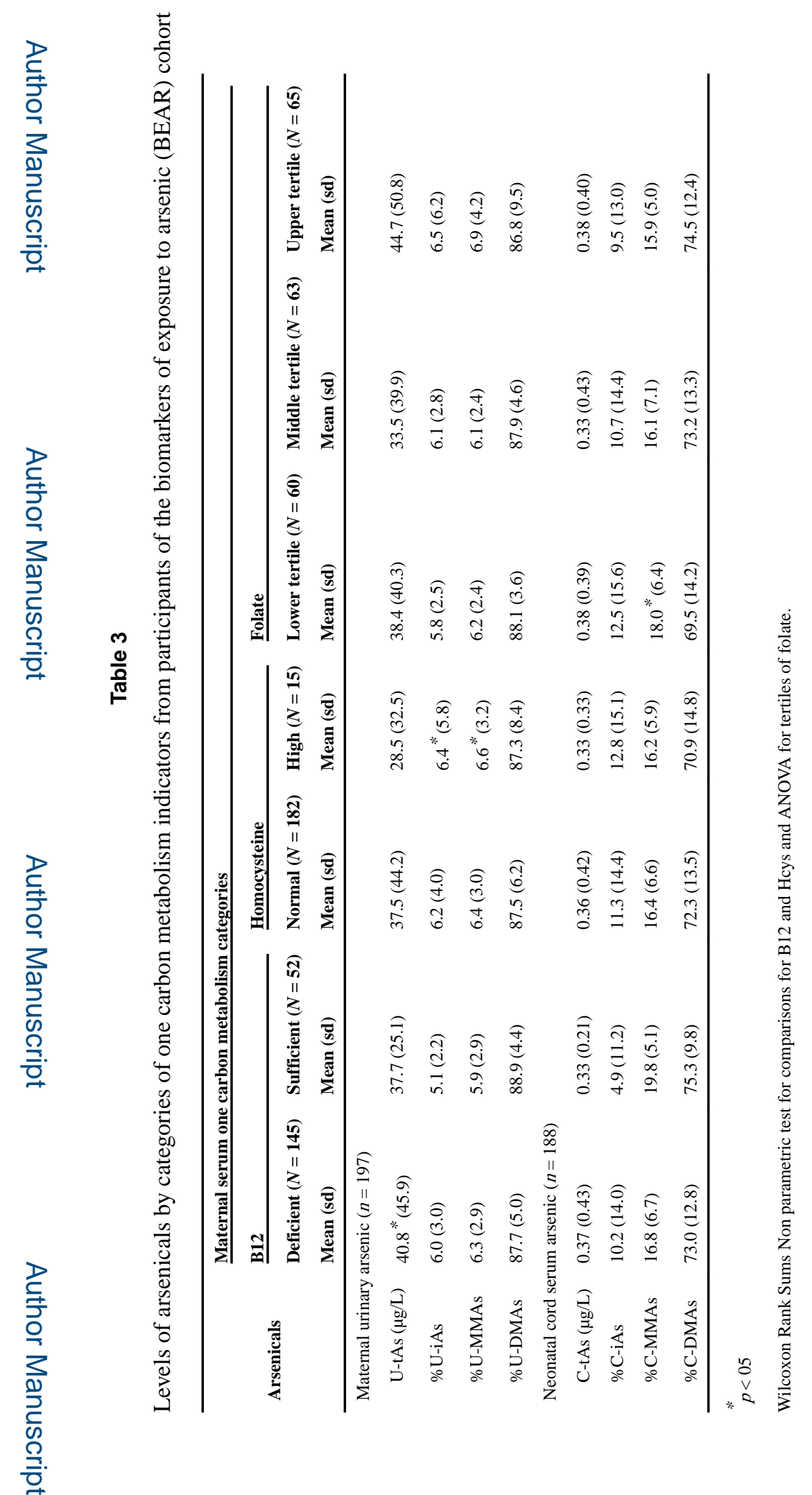




\section{Table 4}

Multivariable $^{b}$ relationships of arsenicals and one-carbon metabolism indicators from participants ${ }^{a}$ of the biomarkers of exposure to arsenic (BEAR) cohort

\begin{tabular}{|c|c|c|c|}
\hline & $\begin{array}{l}\text { B12 } \\
\beta(95 \% \text { CI })[p \text {-value }]\end{array}$ & $\begin{array}{l}\text { Folate } \\
\beta(95 \% \text { CI) [p-value }]\end{array}$ & $\begin{array}{l}\text { Hcys } \\
\beta(95 \% \text { CI })[p \text {-value }]\end{array}$ \\
\hline \multirow[t]{2}{*}{ U-tAs ${ }^{c}$} & -0.56 & 0.027 & 0.44 \\
\hline & $(-0.86,-0.26)[0.0002]^{*}$ & $(-0.22,0.27)[0.82]$ & $(0.043,0.83)[0.029]^{*}$ \\
\hline \multirow[t]{2}{*}{$\% \mathrm{U}-\mathrm{iAs}^{d}$} & 0.037 & -0.098 & -2.1 \\
\hline & $(-0.11,0.18)[0.6]$ & $(-0.44,0.25)[0.57]$ & $(-5.5,1.2)[0.21]$ \\
\hline \multirow[t]{2}{*}{$\%$ U-MMAs $d d$} & 0.088 & 0.097 & -0.29 \\
\hline & $(-0.041,0.22)[0.18]$ & $(-0.20,0.39)[0.51]$ & $(-3.3,2.7)[0.84]$ \\
\hline \multirow[t]{2}{*}{$\%$ U-DMAs $^{d}$} & 1.1 & 0.017 & 1.1 \\
\hline & $(-1.9,4.2)[0.48]$ & $(-0.30,0.33)[0.91]$ & $(-2.0,4.1)[0.48]$ \\
\hline \multirow[t]{2}{*}{ C-tAs ${ }^{c}$} & -0.095 & 0.096 & 0.48 \\
\hline & $(-0.43,0.24)[0.57]$ & $(-0.18,0.37)[0.49]$ & $(0.054,0.91)[0.027]^{*}$ \\
\hline \multirow[t]{2}{*}{$\% \mathrm{C}$-iAs ${ }^{d}$} & 0.21 & -0.025 & -5.0 \\
\hline & $(-0.076,0.49)[0.15]$ & $(-0.77,0.72)[0.94]$ & $(-11.4,1.5)[0.13]$ \\
\hline \multirow[t]{2}{*}{$\%$ C-MMAs ${ }^{d}$} & 0.0562 & -0.17 & 4.4 \\
\hline & $(-0.11, .22)[0.51]$ & $(-0.59,0.25)[0.43]$ & $(0.89,7.9)[0.014]^{*}$ \\
\hline \multirow[t]{2}{*}{$\%$ U-DMAs ${ }^{d}$} & -0.090 & 0.062 & 0.32 \\
\hline & $(-0.27,0.092)[0.32]$ & $(-0.39,0.51)[0.78]$ & $(-3.8,4.4)[0.87]$ \\
\hline
\end{tabular}

${ }^{a}$ Reported results are based upon a sample of 197 and 188, for measured values for urinary (\%U-iAs, \%U-MMAs, \%U-DMAs) and neonatal cord serum arsenicals \%C-iAs, \%C-MMAs, \%U-DMAs), respectively

${ }^{b}$ All models adjusted for maternal age, gestational age, parity, and maternal education

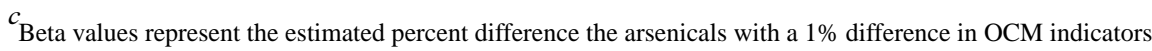

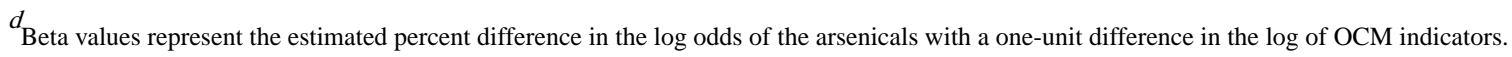

*5\% CI does not include the null of 0

J Expo Sci Environ Epidemiol. Author manuscript; available in PMC 2019 May 23. 